DOI: $10.21105 /$ joss.03394

\section{Software}

- Review ¿

- Repository ca

- Archive ca

Editor: Kevin M. Moerman ־ Reviewers:

- Quellue

- @beniroquai

- @untzag

Submitted: 30 April 2021 Published: 14 August 2021

\section{License}

Authors of papers retain copyright and release the work under a Creative Commons Attribution 4.0 International License (CC BY 4.0).

\title{
ImSwitch: Generalizing microscope control in Python
}

\section{Xavier Casas Moreno ${ }^{1}$, Staffan Al-Kadhimi ${ }^{1}$, Jonatan Alvelid ${ }^{1}$, Andreas Bodén ${ }^{1}$, and Ilaria Testa ${ }^{1}$}

1 SciLifeLab, KTH Royal Institute of Technology

\section{Summary}

The constant development of novel microscopy methods with an increased number of dedicated hardware devices poses significant challenges to software development. On the one hand, software should control complex instruments, provide flexibility to adapt between different microscope modalities, and be open and resilient to modification and extension by users and developers. On the other hand, the community needs software that can satisfy the requirements of the users, such as a user-friendly interface and robustness of the code. In this context, we present ImSwitch, based on the model-view-presenter (MVP) design pattern (Potel, 1996), with an architecture that uses polymorphism to provide a generalized solution to microscope control. Consequently, ImSwitch makes it possible to adapt between different modalities and aims at satisfying the needs of both users and developers. We have also included a scripting module for microscope automation applications and a structure to efficiently share data between different modules, such as hardware control and image processing. Currently, ImSwitch provides support for light microscopy techniques but could be extended to other microscopy modalities requiring multiple hardware synchronization.

\section{Statement of need}

As described in Chhetri et al. (2020), there is a need to have a generalized solution for microscope control, since the usual approach in microscopy labs is to create their own custombuilt software. A complete list is available in Stuurman et al. (2021). ImSwitch is designed to be compatible with many different microscope modalities and customizable to the specific design of individual custom-built microscopes, all while using the same code. We would like to involve the community in further developing ImSwitch in this direction, believing that it is possible to integrate current state-of-the-art solutions into one unified software.

\section{Architecture and implementation}

ImSwitch consists of software modules, e.g., hardware control and image processing, that exchange data through the ModuleCommunicationChannel (Figure 1). Modules can also expose an internal Application Programming Interface (API) to the scripting platform, allowing user-defined scripts to interact with them without requiring the user to modify the code itself to meet their demands. Each of the modules follows the MVP architecture, which has three primary layers: model, view, and presenter. The hardware control module has the following structure: 
- The model layer represents the backend of the program. It has direct access to the hardware through a device sub-layer, which contains the interfaces of the devices, drivers, and Python packages. To provide polymorphism, we have added another sub-layer in the model that consists of what we name managers and multi-managers. The managers are objects that will specify how to control the devices, and which packages should be used. For example, a microscope setup with two cameras and three point detectors will make use of a multi-manager called DetectorsManager, which in turn will manage two instances of CameraManager and three instances of PointDetectorManager. Both managers implement the same functionality through identical function calls but with different implementations.

- The view layer is the Graphical User Interface (GUI). The GUI for each component of the hardware control module is represented by a view element called a Widget. The view layer is linked to the presenter layer by linking each Widget with a WidgetController. The presenters (WidgetControllers) manipulate the views (Widgets) but not vice versa.

- We implemented the presenter layer using two sub-layers: the first sub-layer contains WidgetControllers that mainly read the input of the user through the view and communicate it to the MasterController; the second sub-layer has the MasterCont roller that directly interacts with the managers. The CommunicationChannel serves as a platform to share demands and information between WidgetControllers.

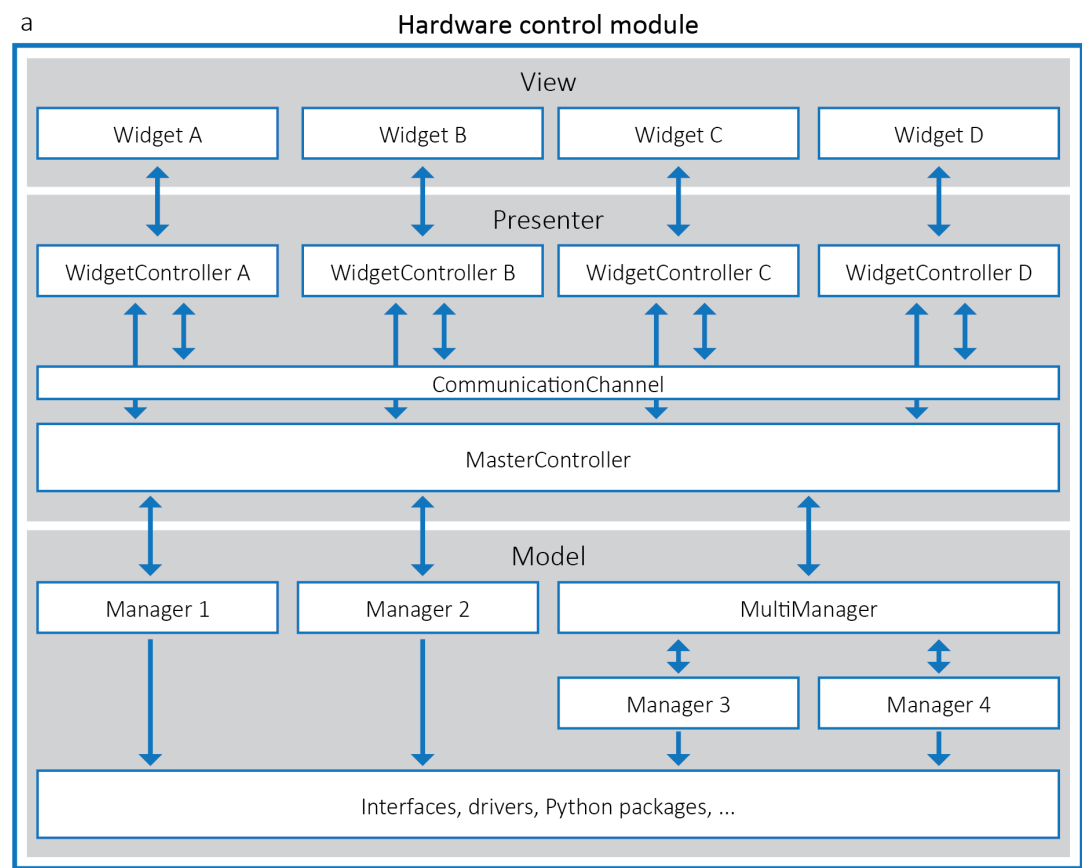

b

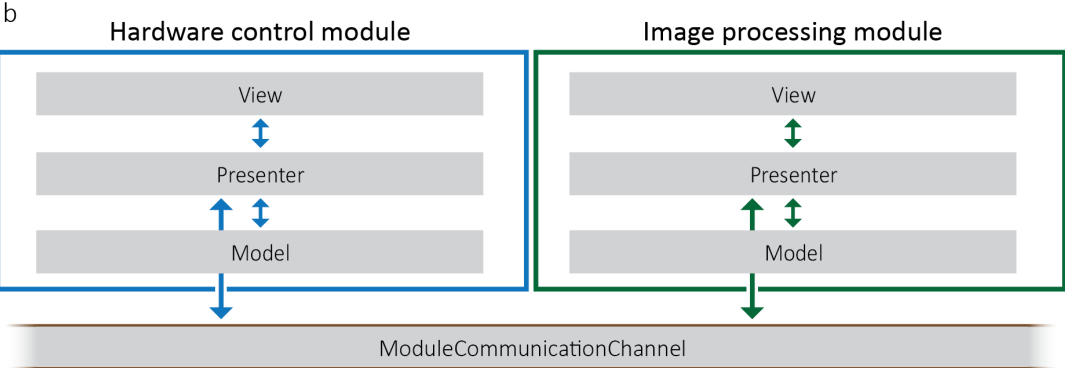

Figure 1: Architecture of ImSwitch 
We use PyQt (PyQT, 2012) for the GUI layout, thread management, and event management; Napari (napari contributors, 2019) for image visualization, which can easily integrate multiple image layers and supports plugins for image processing; and HDF5 (The HDF Group, 20002010) for storing image data and metadata, including all parameters of each experiment and acquisition. The user can readily load metadata from stored files into ImSwitch, allowing experiment acquisition from templates.

Central to the concept of allowing modularity is the use of JSON configuration files to define different microscopes. They contain information about the hardware devices and their connections, and the GUI tools to be loaded.

As a proof of concept ImSwitch has been implemented to control the various microscopes of our lab: point-scanning STED/confocal (Alvelid \& Testa, 2019) and parallelized RESOLFT/confocal (MoNaLISA) (Masullo et al., 2018). These microscopes were previously controlled with a combination of purpose-built software that were microscope-specific or closed-source software. The reader can find more details about the implementations and documentation of ImSwitch at imswitch.readthedocs.io. We have implemented the image processing module for reconstructing MoNaLISA images using our pre-existing shared library. Other microscope modalities requiring designated reconstruction or processing could implement separate modules, using the ModuleCommunicationChannel to retrieve the data, and readily load them into $\mathrm{ImSwitch}$.

\section{Comparison to other software}

$\mu$ Manager (Edelstein et al., 2010) is a free and open-source software for microscope control written in Java and $\mathrm{C}++$, with pervasive driver support to different devices and widely used by microscopists. However, $\mu$ Manager does not adapt fully to complex microscopes that require scanning and triggering or other intricate operations. Moreover, software modification and extension are difficult due to the programming languages and the architecture. Python bindings were recently made available for MMCore, which is the device control layer of $\mu$ Manager, making it compatible with ImSwitch implementation (through the model device sub-layer). Pycro-Manager (Pinkard et al., 2021) provides access to $\mu$ Manager from Python and combines it with image processing and multiple applications. However, custom plugins must still be written for the $\mu$ Manager Java layer, making it complicated to use in complex devices without a pre-existing specialized implementation. This limits the user-friendliness for microscope users.

Microscope (Pinto et al., 2021) is a recent alternative for providing a resilient device layer in Python for controlling microscope devices. It is possible to script microscope experiments and use Cockpit (Phillips et al., 2021) as a GUI. However, the GUI is still limited to simpler microscopes. The most substantial focus of Microscope is to provide a standard interface to control microscope device types. Therefore, it could be integrated with ImSwitch to work towards software generalization by increasing compatibility and including even more devices.

TANGO Controls (Tango Controls, 2015) is a software solution for distributed control systems that provides a communication protocol and an object-oriented architecture for controlling devices of a range of applications. Similar to TANGO Controls, EPICS (Dalesio et al., 1991) focuses on supporting applications that operate complex devices such as particle accelerators and telescopes. While their design and architecture are conceptually similar to ImSwitch, our focus resides on microscopy applications and, in particular, image-based control systems and related hardware when performing experiments and handling data (image acquisition, reconstruction, and analysis). We also provide general tools for microscope users and builders of custom-made microscopes. 


\section{Acknowledgements}

We would like to acknowledge the authors of Tempesta, a further development of Tormenta (Barabas \& Masullo, 2016), for their contributions to earlier software and inspiration for a userfriendly interface. We are also thankful to Francesca Pennacchietti for testing and providing feedback.

We thank the Swedish Foundation for Strategic Research (SSF) funding FFL15-0031 for supporting the project.

\section{References}

Alvelid, J., \& Testa, I. (2019). Stable stimulated emission depletion imaging of extended sample regions. Journal of Physics D: Applied Physics, 53. https://doi.org/10.1088/ $1361-6463 / a b 4 c 13$

Barabas, F., \& Masullo, L. A. (2016). Note: Tormenta: An open source Python-powered control software for camera based optical microscopy. Review of Scientific Instruments, 87(126103). https://doi.org/10.1063/1.4972392

Chhetri, R., Preibisch, S., \& Stuurman, N. (2020). Software for microscopy workshop white paper. http://arxiv.org/abs/2005.00082

Dalesio, L. R., Kozubal, A. J., \& Kraimer, M. R. (1991). EPICS architecture. https://www. osti.gov/biblio/6110347

Edelstein, A., Amodaj, N., Hoover, K., Vale, R., \& Stuurman, N. (2010). Computer control of microscopes using âpmanager. Current Protocols in Molecular Biology, 92(1), 14.20.114.20.17. https://doi.org/10.1002/0471142727.mb1420s92

Masullo, L. A., Bodén, A., Pennacchietti, F., Coceano, G., Ratz, M., \& Testa, I. (2018). Enhanced photon collection enables four dimensional fluorescence nanoscopy of living systems. Nat Commun, 9(3281). https://doi.org/10.1038/s41467-018-05799-w

napari contributors. (2019). napari: a multi-dimensional image viewer for python. https: //doi.org/doi:10.5281/zenodo.3555620

Phillips, M. A., Pinto, D. M. S., Hall, N., Mateos-Langerak, J., Parton, R. M., Titlow, J., Stoychev, D. V., Parks, T., Pinto, T. S., Sedat, J. W., Booth, M. J., Davis, I., \& Dobbie, I. M. (2021). Microscope-cockpit: Python-based bespoke microscopy for bio-medical science. bioRxiv. https://doi.org/10.1101/2021.01.18.427178

Pinkard, H., Stuurman, N., Ivanov, I. E., Anthony, N. M., Ouyang, W., Li, B., Yang, B., Tsuchida, M. A., Chhun, B., Zhang, G., Mei, R., Anderson, M., Shepherd, D. P., Hunt-Isaak, I., Dunn, R. L., Jahr, W., Kato, S., Royer, L. A., Thiagarajah, J. R., ... Waller, L. (2021). Pycro-manager: Open-source software for customized and reproducible microscope control. Nature Methods, 18(3), 226-228. https://doi.org/10.1038/ s41592-021-01087-6

Pinto, D. M. S., Phillips, M. A., Hall, N., MateosLangerak, J., Stoychev, D., Pinto, T. S., Booth, M. J., Davis, I., \& Dobbie, I. M. (2021). Python-microscope: High performance control of arbitrarily complex and scalable bespoke microscopes. bioRxiv. https://doi. org/10.1101/2021.01.18.427171

Potel, M. (1996). MVP: Model-view-presenter the taligent programming model for $c++$ and java.

PyQT. (2012). PyQt reference guide. http://www.riverbankcomputing.com/static/Docs/ PyQt4/html/index.html 
JesS

Stuurman, N., Preibisch, S., Babcock, H., \& Chhetri, R. (2021). uScopeControl (Version 0.1). Zenodo. https://doi.org/10.5281/zenodo.4433237

Tango Controls. (2015). Tango controls. https://www.tango-controls.org

The HDF Group. (2000-20102000-2010). Hierarchical data format version 5. http://www. hdfgroup.org/HDF5 